\title{
A Method to Obtain Complex Transfer Functions of Four Order DC-DC Converters
}

\author{
Josep M. Valls Martí and Pedro M. Gil Izco \\ Department of Electrical and Electronic Engineering, Public University of Navarra (UPNA), Campus of Arrosadía-Pamplona, \\ Navarra 31006, Spain
}

Received: July 20, 2016 / Accepted: August 02, 2016 / Published: October 31, 2016.

\begin{abstract}
Two TFs (transfer functions) are needed to analyze switching DC-DC converters in control-voltage mode: the duty-cycle to output-voltage (control to output) and the input-voltage to output-voltage (line to output). To obtain these TFs a small-signal analysis is required. The CCM (continuous conduction mode) and the DCM (discontinuous conduction mode) analysis are different. When a circuit includes the loss resistances of the components, the number of parameters increases considerably, making manual nodal-loop circuit analysis techniques impractical to obtain the TFs. Moreover, these circuits are bilinear (non-linear) and it is necessary to linearize the equations at a DC operating-point (approximate linearization). Vorpérian describes a PWM (pulse-width-modulated) switch model that includes all non-linear parts of the DC-DC switching converters. This model can be linearized and replaced on the switching converter schematic leading to a linear circuit. At this point it is possible to use symbolic analysis programs to obtain these TFs or to simply apply numerical values for either the Bode diagrams or the calculation of poles and zeros. Here we describe an application of Ekrem Cengelci's method on X DC-DC converter to obtain control to output and line to output TFs in CCM and DCM including loss resistances. The method presented in this paper is optimized to use in the online publishing platform OctaveRS. Also the control to output TF for PCC (peak current controlled) in CCM is obtained.
\end{abstract}

Key words: Ćuk, SEPIC (single ended primary inductance converter), Zeta, X DC-DC converters, mathematics symbolic computation.

\section{Introduction}

Two TFs (transfer functions) are needed to both: analyze the dynamic behavior of four order switching DC-DC converters in control-voltage mode and PCC (peak current controlled) mode.

To this end, it is necessary to apply a symbolic computational method to obtain these TFs [1], because the models used to find them in the CCM (continuous conduction mode) and in the DCM (discontinuous conduction mode) are very complex for manual manipulations [2], especially when the loss resistances of the reactive components are included in order to obtain realistic TFs for these converters.

The Vorpérian PWM switch model [3-6] is used with the (free) circuit-based symbolic manipulation software

Corresponding author: Josep M. Valls Martí, Ph.D. student, research fields: power converters, control, $\mu \mathrm{C}$ and FPGA.
SAPWIN (symbolic analysis program for windows) [7]. The GNU Octave has been used to solve a numerical application with pole-zero analysis and Bode plots. These results are available online at OctaveRS [8], a web platform running GNU Octave [9].

As an example, this method is applied to the $\mathrm{X}$ DC-DC converter [10-12] in order to obtain the duty-cycle to output voltage (control to output) and the input-voltage to output-voltage (line to output) TFs in CCM and in DCM including loss resistances and control to output TF for PCC in CCM.

\section{X-Converter}

As it is pointed in the introduction, the topology studied is the X DC-DC switching converter [12]. This converter shown in Fig. 1 is a four order topological variation of buck-boost converter with Ćuk-class structure. 


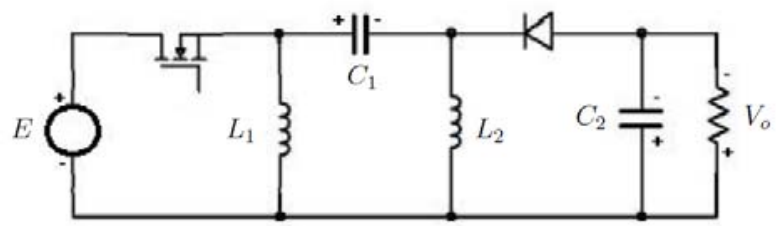

Fig. 1 Power stage of $X$ converter.

Referring to the Ćuk-like class we can say that it is a four order converter, with only one primary switch (MOSFET) and one secondary switch (DIODE). Furthermore, it also has two inductors, one bypass capacitor between input and output stage and one output capacitor.

The main representative circuit of this class is the Ćuk converter, presented in PESC' 77 by Slodoban Cuk [13]. In the same conference, R. P. Massey, described SEPIC (single ended primary inductance converter) [14]. Twelve years later Józwik and Kazimierczuk [15] wrote on Zeta converter. The first description of $\mathrm{X}$ converter is found in Ref. [10], specifically in chapter 1 of the book "Converter Topologies", where the authors Siew-Chong Tan and Chi Kong Tse, called it the "missing" converter. Another reference of this converter is Ref. [11], in this paper Barry W. Williams analyzes several topologies and hear the $\mathrm{X}$ converter is named "New Q5".

In regard with the dynamical modelling of this class of converters, the ideal model dynamic is unrealistic: The lack of loss resistances does not give a right vision of the real behaviour of its dynamics, which usually is to be much more damped than the ideal one. The $\mathrm{X}$ converter schematic that includes the loss resistances is shown in Fig. 2.

To obtain the dynamical averaged model as it is proposed in Ref. [6] it is necessary to substitute the switches by a current and a voltage controlled continuous functions sources that exhibit the same behavior in average state as the initial switches of circuit. For this purpose, this circuit must be redrawn in order to identify (a)-active, (p)-passive and (c)-common terminals as shown in Fig. 3.

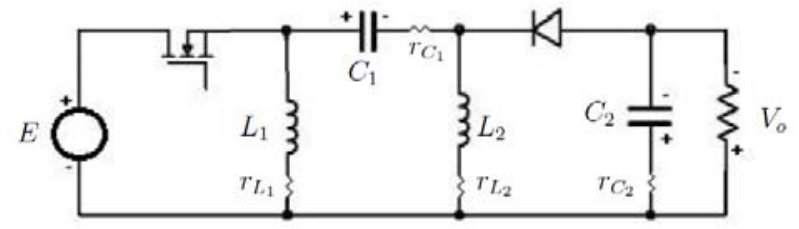

Fig. 2 Power stage of $X$ converter with loss resistances.

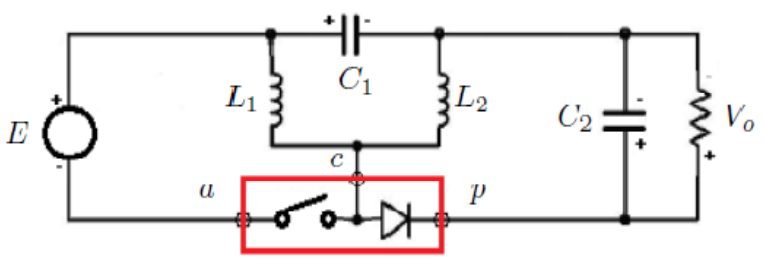

Fig. $3 \mathrm{X}$ converter power stage reordered.

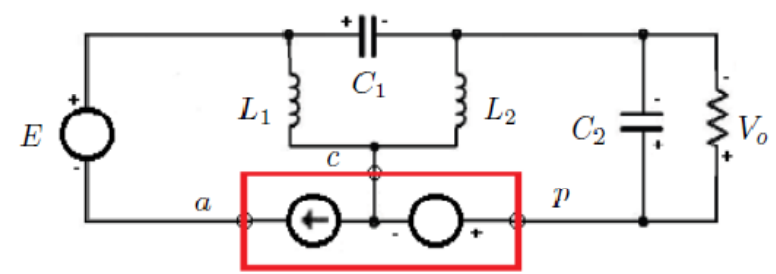

Fig. 4 X converter with PWM-switch model.

Afterwards, as it is mentioned above, the switching devices of the PWM-switch model have to be substituted by a controlled current and voltage sources which represent the average behaviour of the transistor and diode. The final schematic result is shown in Fig. 4.

\section{PWM Switch Model}

Once the PWM switch model is obtained it is necessary to consider that the equations, which define the behavior of the average model of the switch, are different functions whether the mode of operation is in the CCM or DCM. The boundary between CCM and DCM [16] can be determined by Eq. (1) that relates the amplification factor between both modes:

$$
K_{c}=(1-D)^{2}\left\{\begin{array}{l}
\text { if } \frac{2\left(L_{1} \| L_{2}\right)}{R T_{S W}}<K_{c}=>D C M \\
\text { if } \frac{2\left(L_{1}|| L_{2}\right)}{R T_{S W}}>K_{c}=>C C M
\end{array}\right.
$$

The expressions of the equations for the current and voltage controlled sources in CCM and DCM are given respectively by system Eqs. (2) and (3). 


$$
\begin{gathered}
i_{C C M}(t)=i_{a}(t)=i_{c}(t) d(t) \\
v_{C C M}(t)=v_{c p}(t)=v_{a p}(t) d(t) \\
i_{D C M}(t)=i_{a}(t)=i_{p}(t) \mu(t) \\
v_{D C M}(t)=v_{c p}(t)=v_{a c}(t) \mu(t)
\end{gathered}
$$

These non-linear equations are bilinear because they are, independently, linear in the control $(d(t), \mu(t))$ and linear in the state variables $(i(t), v(t))$, but not in both. The non-linear condition is given by the products of state and control variables like $i(t)$ and $d(t)$. To obtain the TFs, previously, it is necessary to linearize the equations at a DC operating-point (approximate linearization). The results of this linearization for CCM and DCM are shown in Eqs. (4) and (5) respectively.

$$
\begin{aligned}
\hat{\imath}_{a}(t) & =I_{c} \hat{d}(t)+D \hat{\imath}_{c}(t) \\
& =S I \hat{d}(t)+D \hat{\imath}_{L_{1}}(\mathrm{t})+D \hat{\imath}_{L_{2}}(\mathrm{t}) \\
\hat{v}_{c p}(t)= & V_{a p} \hat{d}(t)+D \hat{v}_{a p}(t) \\
= & S V \hat{d}(t)+D \hat{e}(\mathrm{t})-D \hat{v}_{C_{1}}(\mathrm{t}) \\
& \quad+\mathrm{D} \hat{v}_{o}(t)
\end{aligned}
$$

Note that $V_{a p}=E+V_{o}=S V$ is the sum of the input and the output voltages, and $I_{C}=I_{L_{1}}+I_{L_{2}}=$ $I_{i}+I_{o}=S I$ is the sum of the input and the output currents.

$$
\begin{aligned}
\hat{\imath}_{a}(t) & =g_{i} \hat{v}_{a c}(t)+k_{i} \hat{d}(t) \\
& =g_{i} \hat{e}(t)+k_{i} \hat{d}(t) \\
\hat{\imath}_{p}(t) & =g_{f} \hat{v}_{a c}(t)+k_{o} \hat{d}(t)-g_{o} \hat{v}_{c p}(t) \\
& =g_{f} \hat{e}(t)+k_{o} \hat{d}(t)-g_{o} \hat{v}_{o}(t)
\end{aligned}
$$

where,

$$
\begin{gathered}
g_{i}=\frac{I_{a}}{V_{a c}}=\frac{\mu^{2}}{R} \\
k_{i}=\frac{2 I_{a}}{D}=\frac{2 \mu V_{o}}{D R} \\
g_{f}=\frac{2 I_{p}}{V_{a c}}=\frac{2 \mu}{R} \\
g_{o}=\frac{I_{p}}{V_{c p}}=\frac{1}{R} \\
k_{o}=\frac{2 I_{p}}{D}=\frac{2 V_{o}}{D R}
\end{gathered}
$$

and taken into account that

$$
\begin{gathered}
V_{c p}=V_{o} \\
I_{p}=I_{o}=\frac{V_{o}}{R}
\end{gathered}
$$

$$
\begin{gathered}
\mu=\frac{V_{o}}{E}=\sqrt{\frac{R T_{S W} D^{2}}{2\left(L_{1} \| L_{2}\right)}} \\
V_{a c}=E \\
I_{a}=I_{i}=\mu I_{p} \\
D=\sqrt{\frac{2\left(L_{1} \| L_{2}\right) \mu^{2}}{R T_{S W}}}
\end{gathered}
$$

The relationship between input and output voltage in CCM is proportional to the duty-cycle and is given by :

$$
V_{o}=\frac{D}{1-D} E-r p_{o} I_{o}
$$

where, $r p_{o}$ is:

$$
\begin{aligned}
r p_{o}=\frac{D^{2}}{(1-D)^{2}} & \left(r_{L_{1}}-(1-D) r_{C_{1}}\right) \\
& +\left(r_{L_{2}}+D r_{C_{1}}\right)
\end{aligned}
$$

These equations are applied to obtain the output voltage, the input and output currents and the rest of the values that are necessary in numerical calculations.

\section{Symbolic Analysis in SAPWIN}

The next step is to introduce the linear equations in graphic mode using the circuit schematic capture in SAPWIN. The control to output TF is obtained by eliminating from the circuit the input voltage source and maintaining duty-cycle effects, as shown in Fig. 5.

The line to output TF is obtained by eliminating from the circuit the duty-cycle source and maintaining the input voltage effects, as shown in Fig. 6.

Fig. 7 shows the schematic of the $\mathrm{X}$ converter used to obtain the control to output TF in DCM.

Fig. 8 shows the schematic of the $\mathrm{X}$ converter used to obtain the line to output TF in DCM.

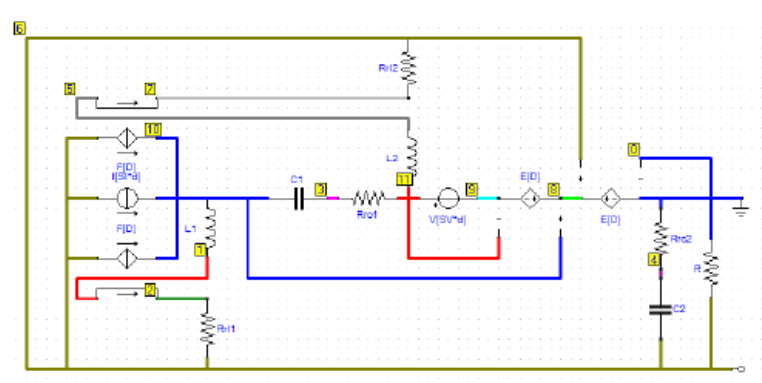

Fig. 5 Control to output TF of the $\mathrm{X}$ converter in CCM. 


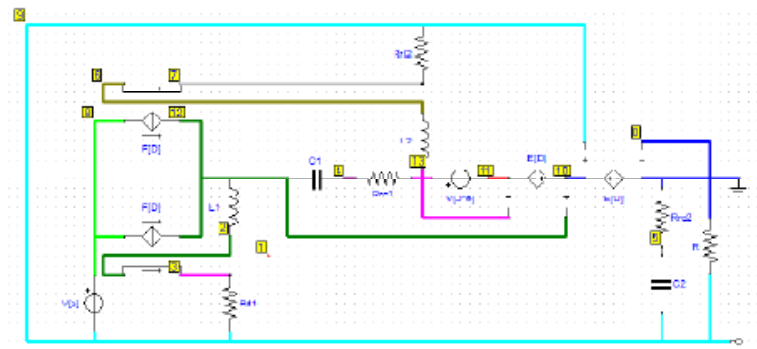

Fig. 6 Line to output TF of the X converter in CCM.

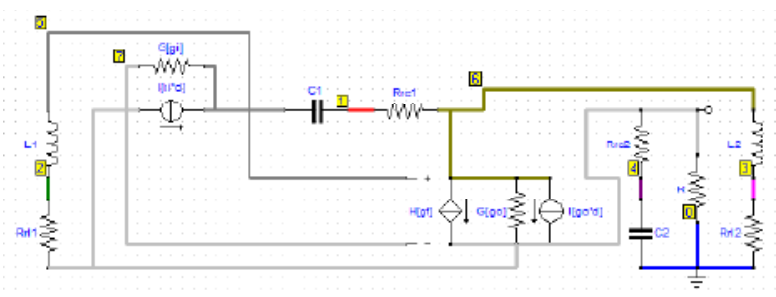

Fig. 7 Control to output TF of the X converter in DCM.

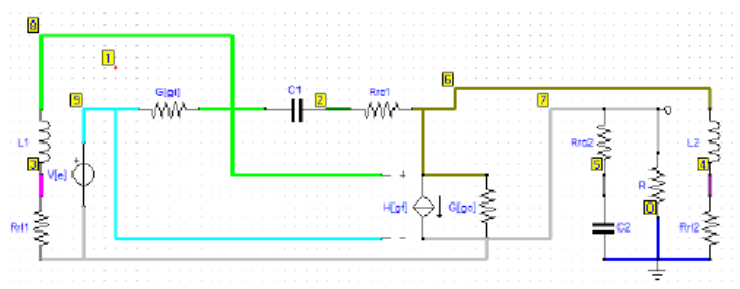

Fig. 8 Line to output TF of the X converter in DCM.

The symbolic expression of the TF is obtained automatically with SAPWIN in a very short space of time, so it allows to skip a process of several tedious hours as would have been necessary if it had been obtained by hand. In a final step, this expression is post-processed by a software developed by the authors to adapt to GNU Octave program through a script file.

\section{Numerical Analysis in OctaveRS}

To obtain the Bode plots of the TF we apply values and execute the script in OctaveRS. These values are the same of an example presented in page 430 of Ref. [17], where it is applied to an SEPIC converter. SEPIC and X belong to the Ćuk-like class converters $[12]$ and the results are comparable.

$$
\begin{array}{ll}
\mathrm{E}=120 \mathrm{~V} & \mathrm{D}=0.4 \\
\mathrm{~L}_{1}=500 \mu \mathrm{H} & \mathrm{L}_{2}=100 \mu \mathrm{H} \\
\mathrm{C}_{1}=47 \mu \mathrm{F} & \mathrm{C}_{2}=200 \mu \mathrm{F} \\
\mathrm{rl}_{1}=0.10 \Omega & \mathrm{rl}_{2}=0.02 \Omega \\
\mathrm{rc}_{1}=0.00 \Omega & \mathrm{rc}_{2}=0.00 \Omega
\end{array}
$$

The load resistance for CCM is $\mathrm{R}_{\mathrm{CCM}}=40 \Omega$ and for $\mathrm{DCM}$ is $\mathrm{R}_{\mathrm{DCM}}=50 \Omega$. In this conditions, the operating point of the converter in CCM is given by:

$$
\begin{aligned}
& V_{o}=79.87 \mathrm{~V}, I_{i}=1.33 \mathrm{~A}, \\
& I_{o}=2.00 \mathrm{~A}, r p_{o}=0.06 \Omega .
\end{aligned}
$$

These other values are calculated for DCM.

$$
V_{o}=83.14 \mathrm{~V}, I_{i}=1.15 \mathrm{~A}, I_{o}=1.66 \mathrm{~A}
$$

With these values the control to output TF in CCM is given by:

$\frac{V_{o}(s)}{d(s)}=\frac{-3.13 \times 10^{-10} s^{3}+0.14 \times 10^{-3} s^{2}+0.38 \times 10^{-3} s+4796}{1.88 \times 10^{-14} s^{4}+9.87 \times 10^{-12} s^{3}+1.34 \times 10^{-6} s^{2}+0.38 \times 10^{-3} s+14.42}$

The poles and zeros of this TF are:

$$
\begin{gathered}
p_{0}=-111.7+7594.2 i \\
p_{1}=-111.7-7594.2 i \\
p_{2}=-150.8+3643.8 i \\
p_{3}=-150.8-3643.8 i \\
z_{0}=4.3141 e+05+0.0000 e+00 i \\
z_{1}=-4.2472 e+01+5.9536 e+03 i \\
z_{2}=-4.2472 e+01-5.9536 e+03 i
\end{gathered}
$$

And in DCM is given by:

$\frac{V_{o}(s)}{e(s)}=\frac{-6.75 \times 10^{-6} s^{2}+0.26 \times 10^{-2} s+1.80}{1.35 \times 10^{-15} s^{4}+2.83 \times 10^{-10} s^{3}+2.20 \times 10^{-7} s^{2}+0.01 s+2.00}$

The poles and zeros of this TF are:

$$
\begin{gathered}
p_{0}=-2.0938 e+05+0.0000 e+00 i \\
p_{1}=-2.0441 e+02+6.6189 e+03 i \\
p_{2}=-2.0441 e+02-6.6189 e+03 i \\
p_{3}=-1.6192 e+02+0.0000 e+00 i \\
z_{0}=749.69 \\
z_{1}=-356.29
\end{gathered}
$$

The Bode plots of control to output TFs are shown in Fig. 9 for CCM (blue line) and DCM (red line).

The line to output TF for CCM comes from:

$$
\frac{V_{o}(s)}{d(s)}=\frac{2.71 \times 10^{-7} s^{2}+5.41 \times 10^{-5} s+9.6}{1.88 \times 10^{-14} s^{4}+9.87 \times 10^{-12} s^{3}+1.34 \times 10^{-6} s^{2}+0.38 \times 10^{-3} s+14.42}
$$

The poles and zeros of this TF are:

$$
\begin{aligned}
& p_{0}=-111.7+7594.2 i \\
& p_{1}=-111.7-7594.2 i \\
& p_{2}=-150.8+3643.8 i \\
& p_{3}=-150.8-3643.8 i \\
& z_{0}=-100.0+5954.1 i \\
& z_{1}=-100.0-5954.1 i
\end{aligned}
$$



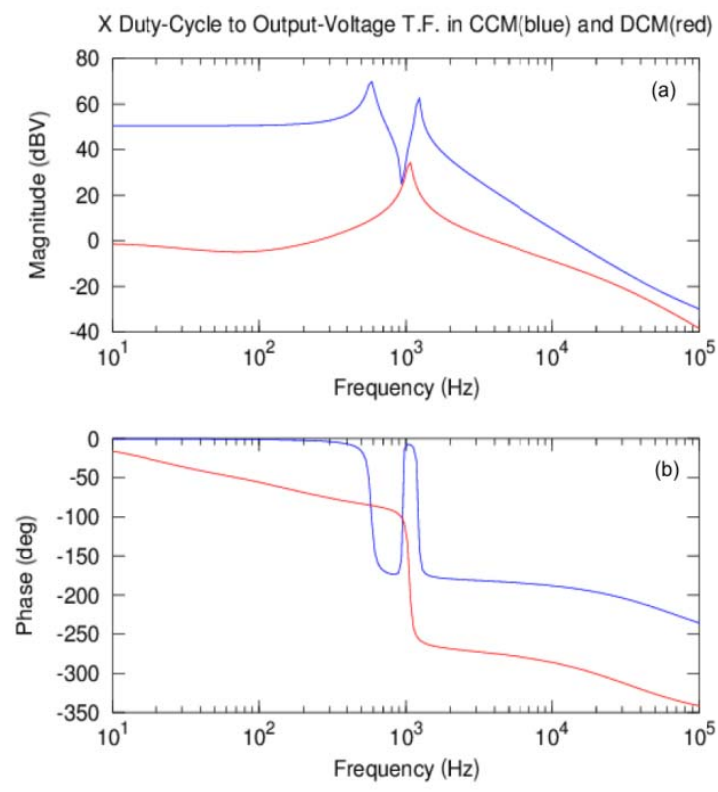

Fig. 9 Bode plot of control to output TF.
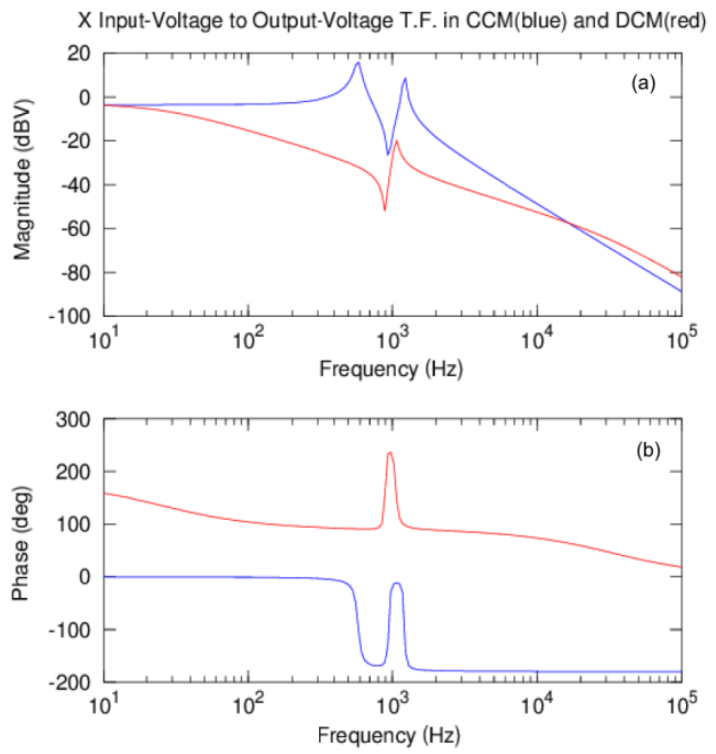

Fig. 10 Bode plot of line to output TF.

And for DCM is given by:

$\frac{V_{o}(s)}{e(s)}=\frac{-4.38 \times 10^{-8} s^{2}-8.77 \times 10^{-6} s+1.39}{1.35 \times 10^{-15} s^{4}+2.83 \times 10^{-10} s^{3}+2.20 \times 10^{-7} s^{2}+0.01 s+2.00}$

The poles and zeros of this TF are:

$$
\begin{aligned}
p_{0}= & -2.0938 e+05+0.0000 e+00 i \\
p_{1}= & -2.0441 e+02+6.6189 e+03 i \\
p_{2}= & -2.0441 e+02-6.6189 e+03 i \\
p_{3}= & -1.6192 e+02+0.0000 e+00 i \\
& z_{0}=-100.0+5620.9 i \\
& z_{1}=-100.0-5620.9 i
\end{aligned}
$$

The Bode plots of line to output TFs are shown in Fig. 10 for CCM (blue line) and DCM (red line).

As always, the control to output and the line to output TFs in CCM have the same poles. The same happens in DCM, as the characteristic equation only depends on the conduction mode.

Moreover, it is relevant to highlight that in all these TFs the poles are negative, which means that the system is asymptotically stable. Also, in the case of the control to output TF, there is at least one positive zero which means this TF is non-minimal phase.

The example covered in this paper is published online at this link:

The example covered in this paper is published online as Ref. [18]. The examples on Ćuk, SEPIC and Zeta are found in Refs. [19-21].

\section{Peak Current Control}

Most control circuits use the peak current control instead of the single output voltage control loop to improve the dynamic behavior of the controlled converter.

The PCC (peak current control) schematic is shown in Fig. 11.

Vorpérian presents a PWM switch model for PCC in CCM [5]. The schematic of this model, linearized for small-signal analysis is shown in Fig. 12.

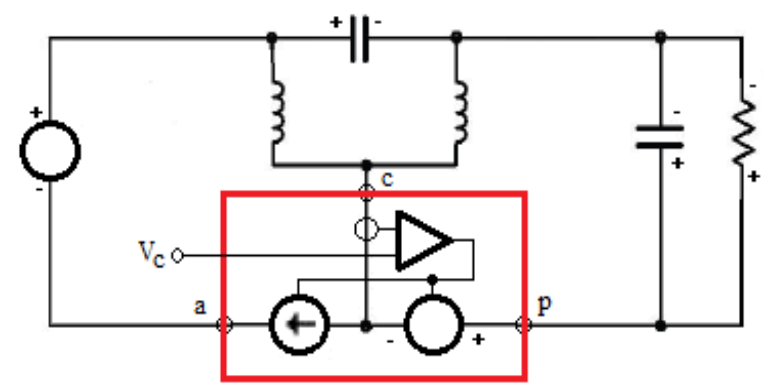

Fig. 11 PCC for X converter schema.

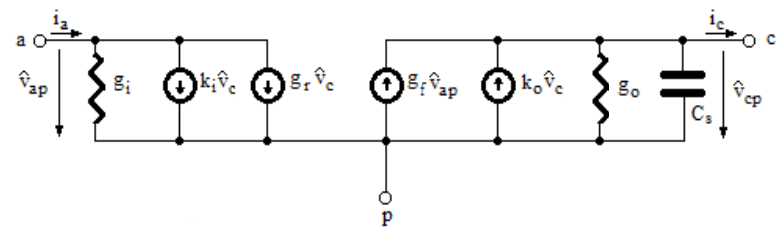

Fig. 12 PWM switch model for PCC in CCM. 
where,

$$
\begin{gathered}
g_{o}=\frac{T_{S W}}{L_{1} \| L_{2}}\left((1-D) \frac{m_{a}}{m_{1}}+\frac{1}{2}-D\right) \\
g_{f}=D g_{o}-\frac{D(1-D) T_{S W}}{2\left(L_{1} \| L_{2}\right)} \\
g_{i}=D\left(g_{f}-\frac{I_{c}}{V_{a p}}\right)=D\left(g_{f}-\frac{I_{i}+I_{o}}{E+V_{o}}\right) \\
g_{r}=\frac{I_{c}}{V_{a p}}-g_{o} D=\frac{I_{i}+I_{o}}{E+V_{o}}-g_{o} D \\
k_{i}=\frac{D}{R_{i}} \quad k_{o}=\frac{1}{R_{i}}
\end{gathered}
$$

and taking into account,

$$
\begin{array}{ccc}
V_{a c}=E & V_{a p}=E+V_{o} & V_{c p}=V_{o} \\
I_{a}=I_{i} & I_{p}=I_{o} & I_{c}=I_{i}+I_{o}
\end{array}
$$

The slopes of the current of the inductors are given by

$$
\begin{gathered}
m_{1}=\frac{V_{a c}}{L_{1} \| L_{2}} R_{i}=\frac{E}{L_{1} \| L_{2}} R_{i} \\
m_{2}=\frac{V_{c p}}{L_{1} \| L_{2}} R_{i}=\frac{V_{o}}{L_{1} \| L_{2}} R_{i} \\
m_{a}=\frac{1}{2} m_{2}
\end{gathered}
$$

Note that this expression of slopes is a simplification that considers that the average voltage drop on the bypass capacitor is zero in the X DC-DC converter.

The capacitor $C_{s}$ models the perturbation on the inductor current that should cause a perturbation in $\hat{v}_{c p}$ having a frequency $\omega_{s} / 2$. Hence for parallel resonance we have [5]:

$$
\begin{gathered}
\frac{\omega_{s}}{2}=\frac{1}{\sqrt{L_{e q} C_{s}}}=>C_{s}=\frac{4}{\left(L_{1} \| L_{2}\right) \omega_{s}^{2}} \\
\text { and } \omega_{s}=2 \pi f_{S W}
\end{gathered}
$$

Finally, the parameter $R_{i}$ which appears in the equations represents the sense resistor used to measure the current in the common terminal.

The operating point, in this case, is the same that was used previously in CCM.

With these values the control to output TF in CCM is obtained by:

$\frac{V_{o}(s)}{V_{c}(s)}=\frac{4.57 \times 10^{-17} s^{4}+1.36 \times 10^{-11} s^{3}-6.76 \times 10^{-6} s^{2}-1.84 \times 10^{-5} s+239.6}{2.3 \times 10^{-21} s^{5}+6.8 \times 10^{-16} s^{4}+2.3 \times 10^{-10} s^{3}+1 \times 10^{-7} s^{2}+8 \times 10^{-3} s+2.2}$

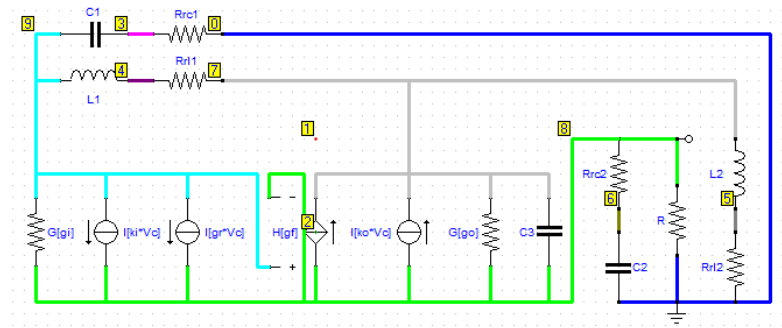

Fig. 13 SAPWIN schematic for X in PCC and CCM.
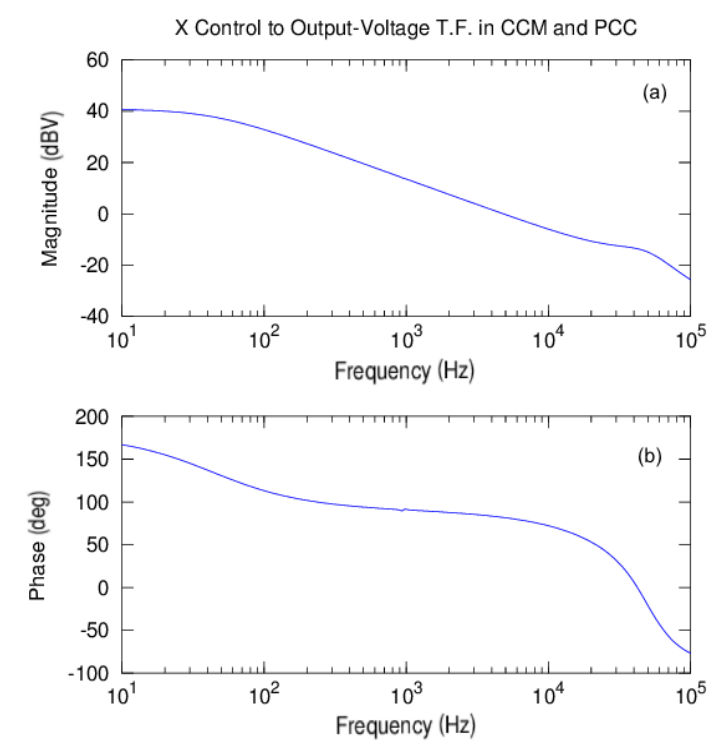

Fig. 14 Bode plot of control to output TF in CCM and PCC.

The poles and zeros of this TF are:

$$
\begin{gathered}
p_{0}=-1.4788 e+05+2.7730 e+05 i \\
p_{1}=-1.4788 e+05-2.7730 e+05 i \\
p_{2}=-3.9619 e+01+5.9527 e+03 i \\
p_{3}=-3.9619 e+01+5.9527 e+03 i \\
p_{4}=-2.7137 e+02+0.0000 e+00 i \\
z_{0}=-5.6007 e+05+0.0000 e+00 i \\
z_{1}=2.6397 e+05+0.0000 e+00 i \\
z_{2}=-3.6842 e+01+5.9527 e+03 i \\
z_{3}=-3.6842 e+01-5.9527 e+03 i
\end{gathered}
$$

The Bode plots of control to output TF in CCM and PCC are shown in Fig. 14.

Note that, as is well known, the PCC offers a better controllability than voltage control.

\section{Conclusions}

The circuit-oriented average model analysis allows to obtain the dynamic model straightforwardly using 
to this end the original circuit of the converter. Therefore, we can assert that it is advantageous over other averaging techniques as it does not need to recourse to the use of state space representation of the system in matrix form. It simplifies converter analysis and yields good intuitive understanding of the converter steady-state and dynamic properties.

The use of the SAPWIN program allows to analyze a complex non-linear circuit without carrying out routine and boring algebraic manipulations prone to errors. It also allows you to compare circuits in a way that otherwise would have been very difficult. The graphical characteristic of this method reduces the complexity to a problem of algebra that can be automated.

This methodology is intended for the use of free programs and resources as the program of symbolical analysis SAPWIN. And it is available on OctaveRS, a platform to publish code GNU Octave of Professor Richard Marquez.

\section{Acknowledgement}

The authors would like to thank Professor Richard Marquez for his advices and support in the preparation of this paper and his contribution to the free software world through his web platform OctaveRS.

\section{References}

[1] Cengelci, E. 2008. "Software Makes Transfer Functions More Manageable." Power Electronics Technology 34 (6): 14-21.

[2] Mweene, L. H. 2009. "Cramer's Rule Helps Solve DC-DC Converter Transfer Functions." Power Electronics Technology 35 (7): 14.

[3] Vorpérian, V. 1990. "Simplified Analysis of PWM Using Model of PWM Switch Part I: Continuous Conduction Mode." IEEE Transactions on Aerospace and Electronic Systems 26 (3): 490-6.

[4] Vorpérian, V. 1990. "Simplified Analysis of PWM Converters Using Model of PWM Switch Part II: Discontinuous Conduction Mode." IEEE Transactions on Aerospace and Electronic Systems 26 (3): 497-505.

[5] Vorpérian, V. 1990. "Analysis of Current-Mode Controlled PWM Converters Using of the Model of the Current-Controlled PWM Switch." In Proceedings of the
International Power Conversion Conference, 183-95.

[6] Vorpérian, V. 2004. Fast Analytical Techniques for Electrical and Electronic Circuits. Cambridge: Cambridge University Press.

[7] U. F. Department of Electronics and Telecommunications. 2001. "SAPWIN, Symbolic Analisys Program for Windows." Accessed April 27, 2015. Available: http://cirlab.det.unifi.it/Sapwin/.

[8] Marquez, R. 2014-2016. "OctaveRS-Share Computational Solutions with GNU Octave or R (Statistical Package)." Accessed March 28, 2015. Available: http://cursosing.net/octavers/.

[9] Eaton, J. W. 1993-2015. "Gnu Octave-High-Level Interpreted Languahe for Numerical Computations." Accessed April 4, 2015. Available: https://www.gnu.org/software/octave/.

[10] Vasca, F., and Iannelli, L. 2012. Dinamycs and Control of Switched Electronic Systems, Advanced Perspectives for Modeling, Simulation and Control of Power Converters. London: Springer-Verlag.

[11] Williams, B. W. 2014. "Generation and Analysis of Canonical Switching Cell DC-to-DC Converters." IEEE Transactions on Industrial Electronics 61 (1): 329-46.

[12] Valls, J. 2014. "Analysis and Synthesis of a New Converter to Complete the Class of Cúk, SEPIC, Zeta Converters." In Proceedings of the Annual Seminar on Automation, Industrial Electronics and Instrumentation, Tangier, Morocco, PCT2-1.

[13] Cuk, S., and Midlebrook, R. D. 1977. "A New Optimum Topology Switching DC-to-DC Converter.” In Proceedings of the Power Electronics Specialists Conference, 160-79.

[14] Massey, R. P., and Snyder, E. C. 1977. "High Voltage Single-Ended DC-DC Converter." In Proceedings of the Power Electronics Specialists Conference, 156-9.

[15] Józwik, J., and Kazimierczuk, M. K. 1989. "Dual SEPIC PWM Switching-Mode DC/DC Power Converter." IEEE Transactions on Industrial Electronics 36 (1): 64-70.

[16] Gil, P., Sebastián, J., Cobos, J. A., and Uceda, J. 1992. "The Determination of the Boundaries between Continuous and Discontinuous Conduction as Power Factor Preregulators Modes in PWM DC-to-DC Converters Used." In Proceedings of the Power Electronics Specialists Conference, 1061-70.

[17] Erickson, R. W., and Maksimovic, D. 2001. Fundamentals of Power Electronics. 2nd ed. Boulder, Colorado: Kluwer Academic Publishers.

[18] Valls, J. 2015. "Control Transfer Functions on X DC-DC Converter with Loss Resistances." Accessed January 25, 2016. http://cursosing.net/octavers/codigos/listing/547control-transfer-functions-on-X-dc-dc-converter-with-loss -resistances.

[19] Valls, J. 2015. "Control Transfer Functions on CUK 
DC-DC Converter with Loss Resistances." Accessed January 25, 2016. http://cursosing.net/octavers/codigos/ listing/545-control-transfer-functions-on-cuk-dc-dc-conv erter-with-loss-resistances.

[20] Valls, J. 2015. "Control Transfer Functions on SEPIC DC-DC Converter with Loss Resistances." Accessed January 25, 2016. http://cursosing.net/octavers/codigos/ listing/292-control-transfer-functions-on-sepic-dc-dc-con verter-with-loss-resistances.

[21] Valls, J. 2015. "Control Transfer Functions on Zeta DC-DC Converter with Loss Resistances." Accessed January 25, 2016. http://cursosing.net/octavers/codigos/ listing/546-control-transfer-functions-on-zeta-dc-dc-conv erter-with-loss-resistances. 